\title{
Renaissances and current trends with electrochemical sensors and biosensors
}

\section{Some history of electrochemical sensors}

The history of electrochemical sensors started with the discovery of glass lamellas as $\mathrm{pH}$-sensitive membranes by Cremer in $1906^{1}$ and their characterization as potentiometric devices by Haber in $1909 .{ }^{2}$ In the nineteen twenties the next milestone was laid by Heyrovsky with the invention of "polarography"3 which was later named "voltammetry" leaving the original term only for methods using the dropping mercury electrode (DME). Whereas the subsequent decades were dominated by polarography accompanied by a levelling-off interest in electroanalysis, a first renaissance emerged from the nineteen sixties onward when new measuring techniques were developed (e.g., differential pulse voltammetry) on one hand in combination with stripping analysis on the other. During that period an immense number of publications appeared dealing mainly with the determination of the four "classical" metals easily accessible by inverse voltammetry on mercury electrodes, i.e., $\mathrm{Zn}, \mathrm{Cd}, \mathrm{Pb}$ and $\mathrm{Cu}$. Along with such prosperity in voltammetry also potentiometry slipped into its golden age with the development of new ion selective membranes for many of the elements of the periodic system. In the nineteen eighties a second renaissance could be noticed due to the chemical modification of amperometric electrodes. Whereas the use of mercury electrodes decreased new electrode materials moved into the focus of interest, such as vitreous carbon (glassy carbon), noble metals, heterogeneous composites (carbon paste, ${ }^{4}$ screen-printed electrodes) in combination with altered surfaces which facilitated broader applicability of electroanalytical methods. Earlier landmarks of sensor design, such as the first amperometric sensor (oxygen electrode $^{5}$ ) and the first biosensor (glucose sensor ${ }^{6}$ ), both developed by Leland Clark, contributed to a peak-like increase of electroanalytical studies.

Currently we are experiencing the third renaissance of electroanalysis and electrochemical sensors. Since the start of the new millennium, along with the expansion of nanotechnology, an increasing and massive number of publications describe sensors and biosensors containing nano-sized materials in the recognition layer. Such particles possess specific properties and may be employed in various shapes, compositions and mutual combinations. Nowadays this fashionable habit, sometimes not born by necessity, is even topped and pushed forward by one material - graphene, which seems by now to be the most interesting nano-sized structure of all. In the following paragraphs a few of the current trends will be summarized in short.

\section{Electrode materials}

Due to an almost pandemic mercury phobia the use of this metal as an electrode material has become practically obsolete. Occasionally amalgam electrodes based on silver wires are still in use where the amount of $\mathrm{Hg}$ is at a minimum. Alternatives have been introduced, mainly in the form of film electrodes such as bismuth ${ }^{7}$ and antimony; ${ }^{8}$ but usually they do not cope completely with the outstanding characteristics of mercury. Recently also films of metals with less noble character were described (e.g., lead ${ }^{9}$ ). Diamond is particularly worth of mentioning because it becomes an electrically

\author{
Volume 3 Issue 5 - 2017
}

Kurt Kalcher
Karl-Franzens University, Austria

Correspondence: Kurt Kalcher, Karl-Franzens University, Graz, Universitaetsplatz I,A-80I0 Graz,Austria, Email kurt.kalcher@uni-graz.at

Received: December 01, 2017 | Published: December 2I, 2017

conductive p-semiconductor by doping with boron and shows good electrochemical properties like wide applicable positive potential window and small tendency to adsorb analytes. Due to the ease of even multiple modification under mild conditions (especially important for producing biosensors) heterogeneous electrode formulations are still favorite materials, particularly carbon paste, invented by Adams in 1958 already, ${ }^{4}$ and inks for screen printing. As the latter ones yield mechanically more stable and robust sensors by requiring only small amounts of printing material, they seem most suitable for commercialization and mass production. Disadvantages such as varying electrode characteristics can be compensated by supplying calibration data on a chip which has to be placed into the measurement device by the customer.

\section{Nanomaterials}

Nowadays nanomaterials are extensively used to modify sensor surfaces to obtain improved analytical characteristics. Gold nanoparticles have been in use for quite a long period already and are still in the focus of interest; one important feature is their high ability to chemisorb sulfur-containing compounds, such as thiols and disulfides; as the latter structural elements appear also in enzymes they are a simple platform for their immobilization. Apart from noble metal nanoparticles from oxides and sulfides, composites and organic compounds and polymers are widely used. Core-shell nanoparticles offer a variety of features, such as specific binding of substrates or easy handling due to magnetic properties. ${ }^{10}$ Of special importance are carbon-based nanomaterials, i.e., graphene nanodots, carbon nanotubes and nanoribbons, graphene (which is mostly reduced graphene oxide) and graphene nanoplatelets. Functionalization of graphene and its composites with nanoparticles opens an almost endless avenue for designing electrode modifiers with specific properties and characteristics. Even quantum dots, artificial quasi-atoms with discrete electronic states and fluorescence properties due to electronic space restriction, are used for electrochemical purposes.

Nanomaterials have opened a new dimension for the production of electrochemical sensors, a tendency that will persist at least for some decade due to the enormous variability with respect to size, shape and composition. Nevertheless it can be noticed already that possibly not all applications where nanomaterials are involved display an inherent necessity to use them, but that authors just follow a fashion trend to increase the attractiveness of their work. Additionally, working 
with nanoparticles often involves time-consuming and sophisticated operations which are not very user-friendly in routine analysis where usually a high sample throughput rate is desirable. Commercialization also seems problematic with many sensing systems involving nanoparticles due to a lack of robustness combined with short lifetimes (storage and operation). Apart from this, nanomaterials are becoming emerging environmental pollutants ${ }^{11}$ with the possible consequence of legal actions against their industrial exploitation.

\section{Electrochemical Approaches}

The voltammetric techniques applied to electrochemical sensing are practically the same as they appeared with the former renaissances of electroanalysis. Cyclic voltammetry is still the main assay to characterize electrode surfaces and electrochemical processes often together with electrochemical impedance spectroscopy to quantify the extent of improvement of the charge transfer resistance. Fast scanning techniques, such as linear sweep and square wave voltammetry as well as differential pulse voltammetry are commonly used to record the signals. Amperometric sensing is preferential with devices which are not prone to interferences from the sample matrix. Though measurement out of the equilibrium accompanied by low detection limits has provoked an impact on the development and use of ionselective electrodes, potentiometric detection methods are still in minority compared to amperometric ones.

\section{Analytes}

All possible types of analytes, inorganic or organic, may be assessed by electrochemical methods if they yield a signal either directly or indirectly. Recently there seems to be an increasing interest in inorganic analytes again, for example, in heavy metals. The assays include electrocatalysis, ${ }^{12}$ accumulation via ionic liquids,${ }^{13}$ or enzymatic inhibition. ${ }^{14}$ Evidently sensors for the detection of elements will not compete with laboratory desktop methods (atomic absorption spectroscopy, inductively coupled plasma mass spectrometry). But, because of their simplicity, portability and cost-effectiveness, small hand-held devices will offer the big advantage of providing at least threshold information at the site of interest directly, thus helping with the decision if a sample should be further analyzed or not. Nevertheless, the main concern of electrochemical sensors is organic compounds of environmental and biological interests. Cancer markers are one topic among many others, such as herbicides, pesticides, toxins, alkaloids and drugs. Electrochemical sensors were even used in extraterrestrial applications. ${ }^{15}$ One of the main drawbacks of analyses with electrochemical sensors relying on the measurement of current is the fact that the analyte must have a direct or indirect electrochemical activity within the applied potential window. Another implication refers to the selectivity which can be excellent in standard solutions but fails with complex matrices. A further problem might be the limit of detection, which is in many cases not low enough to provide unambiguous results in biological, medical and environmental analysis.

\section{Biosensors}

Biosensors constitute the main core of literature on electrochemical sensors. Among all these reports enzyme-based sensors are still in majority, especially oxidoreductases according to the chemical nature of amperometric detection. As the active center of enzymes is very often buried deep in the protein shell, direct electron transfer is possible only via wires consisting of redox polymers, nanoparticles or nanoribbons (third generation of oxidase sensors ${ }^{16}$ ). Crude extracts and even tissues as biological receptors circumvent the isolation of pure enzymes, but may produce strongly varying signals due to different contents of the biocatalyst in the source material. Nucleic acids are electroactive (guanine having the lowest oxidation potential) and can be directly determined via sorptive accumulation on carbon paste electrodes. Immobilized double-stranded deoxyribonucleic acid (DNA) may either serve as accumulator for intercalating species or for interaction studies whereas single-stranded DNA may be used for genosensors via hybridization with target strands. Immunosensors with immobilized antibodies show high sensitivity towards the analyte (antigen) in sandwich or competitive assays. Recently aptamer sensors with small oligonucleotides which specifically bind to the target are emerging as concurrents of the antigen-antibody types because they allow (after the release of the counter-strand) amplification cycles to achieve extremely low detection limits. In this respect, molecular polymer imprinting should be also mentioned as some method of mimicking synthetic antibodies where a template (analyte) is bound to a polymer and removed afterwards leaving a cavity which possesses the shape and also specific binding sites for the target. Cytosensors, which are able to recognize and detect specific cells, in particular cancer cells, are usually based on immunological reactions. Biosensors suffer from many drawbacks with respect to routine analysis and commercialization. Many biosensors produced in the laboratory behave like individuals with low reproducibility which inflicts suffering on mass production. Of course, such problems can be technically solved but are usually combined with a high effort in quality control. Another aspect is the shelf-lifetime of biosensors, which is normally quite short due to the sensitivity of biological materials to environmental influences and storage conditions. Thus, quite a lot of research is currently devoted to altering enzymes to make them more stable and less prone to deactivation.

The practical efficiency of genosenors, particularly for detecting viral diseases, is nowadays rather low mainly because the concept suffers from some systemic problems: As oligonucleotides are used the specificity is not high whereas long strands will change over some generations of viral reproduction due to mutation. Immunosensors require quite a few long-lasting handling procedures, particularly if amplification steps are involved, which excludes them for practical routine analysis. Molecular imprinting suffers mainly from specificity. In this respect many procedures and concepts published so far may be regarded as necessary fundamental work, but will never reach the level of usefulness.

\section{Future perspectives}

In the new millenium electrochemical sensors have again attracted enormous interest due to the use of nanoparticulate matter in the receptor sphere of sensors. One main drawback is still the limited shelf life time of biosensors. Much research is done on modifying enzymes and biological components to increase their stability and robustness. On the other hand miniaturized sensors on chips, sensor arrays either for simultaneous determination of analytes or for designing electrochemical noses, tongues and complete micro total analysis systems ( $\mu \mathrm{TAS})$ are very promising tools for advanced intelligent sensing. In robust and stable configurations they will be a valuable aid in decentralized point-of-care testing (POCT). Combinations of implanted sensors with micro-electromechanical systems (MEMS) powered by biofuel cells obtaining their energy from nutrients in the blood will be extremely useful therapeutic approaches in the future. 


\section{Acknowledgements}

None.

\section{Conflict of Interest}

The author declares no conflict of interest.

\section{References}

1. Cremer M. Über die Ursache der elektromotorischen Eigenschaften der Gewebe, zugleich ein Beitrag zur Lehre von polyphasischen Elektrolytketten. Z Biol. 1906;47:562-608.

2. Haber F, Klemensiewicz Z. Über elektrische Phasengrenzflächenkräfte. Z Phys Chem. 1909;67:385-427.

3. Heyrovsky J. Electrolysis with a dropping mercury electrode (in Czech). Chem Listy. 1922;16:258-264.

4. Svancara I, Kalcher K, Walcarius A, et al. Electroanalysis with carbon paste electrodes. USA: CRC Press; 2012. 666 p.

5. Clark Lc Jr, Wolf R, Granger D, et al. Continuous recording of blood oxygen tensions by polarography. J Appl Physiol. 1953;6(3):189-193.

6. Clark LC, Lyons C. Electrochemical Glucose Biosensors. Ann N Y Acad Sci. 1962;102:29-45.

7. Wang J, Lu J, Hocevar SB, et al. Bismuth-coated carbon electrodes for anodic stripping voltammetry. Anal Chem. 2000;72(14):3218-3222.

8. Pauliukaite R, Metelka R, Svancara I, et al. Screen-printed carbon electrodes bulk-modified with $\mathrm{Bi}_{2} \mathrm{O}_{3}$ or $\mathrm{Sb}_{2} \mathrm{O}_{3}$ For trace determination of some heavy metals. Sci Papers Univ Pardubice. 2004;(A)10:47-58.
9. Bobrowski A, Kalcher K, Kurowska K. Microscopic and electrochemical characterization of lead film electrode applied in adsorptive stripping analysis. Electrochim Acta. 2009;54(28):7214-7221.

10. Samphao A, Butmee, Pukahuta C, et al. Flow-injection amperometric determination of glucose using a biosensor based on immobilization of glucose oxidase onto $\mathrm{Au}$ seeds decorated on core $\mathrm{Fe}_{3} \mathrm{O}_{4}$ nanoparticles. Talanta. 2015;142:35-42.

11. Stuart EJE, Compton RG. Nanoparticle-emerging contaminants. Environmental analysis by electrochemical sensors and biosensors: Springer; 2014. p. 855-878.

12. Bobrowski A, Zarebski J. Catalytic adsorptive stripping voltammetry at film electrodes. Current Anal Chem. 2008;4:191-201.

13. Chaiyo S, Mehmeti E, Siangproh W, et al. Non-enzymatic electrochemical detection of glucose with a disposable paper-based sensor using a cobalt phthalocyanine-ionic liquid-graphene composite. Biosens Bioelectron. 2008; 102:113-120.

14. Samphao A, Rerkchai H, Jitcharoen J, et al. Indirect determination of mercury by inhibition of glucose oxidase immobilized on a carbon paste electrode. Int J Electrochem Sci. 2012;7:1001-1010.

15. Mc El Honey KM, O Neil GD, Kounaves SP. Environmental Analysis by Electrochemical Sensors and Biosensors. In: Moretto LM et al. editors. 1st ed. USA: Springer; 2014. 713 p.

16. Mehmeti M, Stanković DM, Chaiyo S, et al. Wiring of glucose oxidase with graphene nanoribbons: an electrochemical third generation glucose biosensor. Microchim Acta. 2017;184(4):1127-1134. 